\title{
Maximum-entropy-type Lyapunov functions for robust stability and performance analysis*
}

\author{
Dennis S. Bernstein \\ Department of Aerospace Engineering. The University of Michigan, Ann Arbor, MI 48109-2140, USA
}

\author{
Wassim M. Haddad \\ Department of Mechanical and Aerospace Engineering, Florida Institute of Technology, Melbourne, FL 32901, USA
}

David C. Hyland

Harris Corporation, Goternment Aerospace Systems Division, MS 22/4847, Melbourne. FL 32902, USA

Feng Tyan

Department of Aerospace Engineering. The University of Michigan, Ann Arbor, MI 48109-2140. USA

Received 30 March 1992

Revised 15 September 1992

\begin{abstract}
We present two Lyapunov functions that ensure the unconditional stability and robust performance of a modal system with uncertain damped natural frequency. Each Lyapunov function involves the sum of two matrices, the first being the solution to the so-called maximum-entropy equation and the second being a constant auxiliary portion. The significant feature of these Lyapunov functions is that the guaranteed robust stability region is independent of the weighting matrix, while the performance bounds are relatively tight compared to alternative approaches. Thus, these Lyapunov functions are less conservative than standard bounds that tend to be highly sensitive to the choice of state space basis.
\end{abstract}

Keywords: Maximum-entropy function; robust stability; robust performance

\section{Introduction}

The maximum-entropy approach to robust control was specifically developed to address the problem of modal uncertainty in flexible structures $[2,5,6,18,19]$. The rationale for this approach was based upon insights from the statistical analysis of lightly damped structures [20]. Despite favorable comparisons to other approaches $[9,10,12,13]$ and experimental application [11], the basis and meaning of the approach remain mostly empirical and largely obscure. The purpose of this paper is to make significant progress in developing a rigorous foundation for this approach.

Besides the statistical modal analysis techniques of [20], a variety of formulations have been put forth for justifying the maximum-entropy approach. To reproduce certain covariance phenomena of uncertain

Correspondence to: D.S. Bernstein, Department of Aerospace Engineering, The University of Michigan. Ann Arbor, MI 48109-2140, USA.

* This research was supported in part by the Air Force Office of Scientific Research under grant F49620-92-J-0127 and contract F49620-91-C-0019, the National Science Foundation under Research Initiation Grant ECS-9109558 and the National Aeronautics and Space Administration under contract NAS8-38575. 
multimodal systems (decorrelation, incoherence, and equipartition; see [20]), a multiplicative white-noise model was invoked $[18,19]$. The specific model chosen was interpreted in the sense of Stratonovich, thus entailing a critical correction term in the covariance equation due to the conversion from Stratonovich to Ito calculus. The Stratonovich model was itself based upon a limiting process in which the parameter entropy increased, thus suggesting the name "maximum-entropy" control. White-noise models as a basis for robust control are discussed in [1].

An alternative justification for the maximum-entropy model was given in [14] in terms of positive real transfer functions. This attempt was motivated by the observation that in the limit of high modal frequency uncertainty the maximum-entropy controller assumed a rate dissipative structure $[18,19]$. An alternative attempt to justify the maximum-entropy model was given in [17], where a covariance averaging approach [16] was used to show that if the state covariance is averaged over uncertain modal frequencies possessing a Cauchy distribution, then the resulting averaged covariance satisfies the maximum-entropy covariance model.

Although the various formulations of maximum-entropy thcory lend considerable insight into the nature of the approach, there remains a significant gap between this approach and more conventional techniques, such as $H_{\infty}$ theory. The missing link, in our opinion, is the lack of a Lyapunov function that guarantees the robust stability of the closed-loop control system. In this regard it was long suspected that such a Lyapunov function would be unconventional, that is, unlike those arising in $H_{x}$ theory. This view arose from the fact that the maximum-entropy controllers were often robust to large perturbations in the damped natural frequencies, that is, the imaginary part of the eigenvalues. Such perturbations are highly structured, and thus are often treated conservatively by conventional small-gain-type bounds.

The goal of the present paper is to provide a Lyapunov function basis for the maximum-entropy covariance model for the case of modal frequency uncertainty. In fact, in this special case, we provide two alternative Lyapunov functions along with the corresponding performance bounds. Each Lyapunov function involves the sum of two matrices, the first being the solution to the maximum-entropy equation (see equation (22)) and the second being a constant auxiliary portion. This construction is similar to the parameterdependent Lyapunov function technique developed in [15] except that in the present paper the auxiliary portion is constant, that is, independent of the uncertainty.

The maximum-entropy equation (22) differs fundamentally from alternative robustness tests such as those given in [3,4]. Specifically, whereas the modified Lyapunov functions in [3] involve additional nonnegativedefinite terms in the Lyapunov equation, the maximum-entropy equation entails an indefinite modification. This distinction appears to play a critical role with respect to the way in which the maximum-entropy equation deals with the change in basis induced by the input and weighting matrices.

While this paper potentially provides a Lyapunov function foundation for the maximum-entropy control approach, our results are limited to open-loop analysis. Future research will focus on robust stability of the closed-loop system for the controllers given in $[2,5,6,9-13,18-20]$. Furthermore, although the techniques used to construct the Lyapunov functions for the maximum-entropy equation are limited to modal frequency uncertainty, they appear to be generalizable to larger classes of uncertainty. Nevertheless, for structures with modal frequency uncertainty $[2,5,6,9-13,18,19]$, these results have practical ramifications.

\section{Robust stability and performance problems}

Let $\mathscr{U} \subset \mathbb{R}^{n \times n}$ denote a set of perturbations $\Delta A$ of a given nominal dynamics matrix $A \in \mathbb{R}^{n \times n}$. It is assumed that $A$ is asymptotically stable and that $0 \in \mathscr{M}$.

Robust stability problem. Determine whether the linear system

$$
\dot{x}(t)=(A+\Delta A) x(t), \quad t \in[0, \infty),
$$

is asymptotically stable for all $\Delta A \in \mathscr{U}$. 
Robust performance problem. For the disturbed linear system

$$
\begin{aligned}
& \dot{x}(t)=(A+\Delta A) x(t)+D w(t), \quad t \in[0, \infty), \\
& z(t)=E x(t),
\end{aligned}
$$

where $w(\cdot)$ is a zero-mean $d$-dimensional white-noise signal with intensity $I_{d}$, determine a performance bound $\beta$ satisfying

$$
\mathscr{T}(\mathscr{U}) \triangleq \sup _{\Delta A \in \mathbb{H}} \limsup _{t \rightarrow \infty} E\left\{\|z(t)\|_{2}^{2}\right\} \leq \beta .
$$

For convenience, define the $n \times n$ nonnegative-definite matrices $R \triangleq E^{\mathrm{T}} E$ and $V \triangleq D D^{\mathrm{T}}$. The following result is immediate. For a proof, see [3].

Lemma 2.1. Suppose $A+\Delta A$ is asymptotically stable for all $\triangle A \in \mathscr{U}$. Then

$$
\mathscr{T}(\mathscr{U})=\sup _{\Delta A \in \nVdash} \operatorname{tr}\left(Q_{\Delta A} R\right)=\sup _{\Delta A \in \sharp} \operatorname{tr}\left(P_{A A} V\right),
$$

where $Q_{\Delta A} \in \mathbb{R}^{n \times n}$ and $P_{\Delta A} \in \mathbb{R}^{n \times n}$ are the unique, nonnegative-definite solutions to

$$
0=(A+\Delta A) Q_{\Delta A}+Q_{\Delta A}(A+\Delta A)^{\mathrm{T}}+V
$$

and

$$
0=(A+\Delta A)^{\mathrm{T}} P_{\Delta A}+P_{\Delta A}(A+\Delta A)+R .
$$

Conditions for robust stability and robust performance are developed in the following theorem. Let $\mathscr{N}^{n}$ and $\mathscr{S}^{n}$ denote the sets of $n \times n$ nonnegative-definite and symmetric matrices, respectively.

Theorem 2.2. Let $\Omega_{0}: \mathscr{V}^{n} \rightarrow \mathscr{S}^{n}$, and suppose there exists $P \in \mathscr{N}^{n}$ satisfying

$$
0=A^{\mathrm{T}} P+P A+\Omega_{0}(P)+R .
$$

Furthermore, let $P_{0}: \mathscr{U} \rightarrow \mathscr{S}^{n}$ and $R_{0} \in \mathscr{S}^{n}$ be such that $R_{0} \leq R$,

$$
\Delta A^{\mathrm{T}} P+P \Delta A \leq \Omega(P, \Delta A)+R_{0}, \Delta A \in \mathscr{U},
$$

and

$$
P+P_{0}(\Delta A) \geq 0, \quad \Delta A \in \mathscr{U},
$$

where

$$
\Omega(P, A A) \triangleq \Omega_{0}(P)-\left[(A+A A)^{\mathrm{T}} P_{0}(A A)+P_{0}(A A)(A+\Delta A)\right] .
$$

Then

$$
\left(R-R_{0}, A+\Delta A\right), \quad \Delta A \in \mathscr{U},
$$

is detectable if and only if

$$
A+\Delta A, \Delta A \in \mathscr{M},
$$

is asymptotically stable. In this case, the following statements are true. If $\gamma<1$ is such that $R_{0} \leq \gamma R$, then

$$
P_{\Delta A} \leq \frac{1}{1-\gamma}\left(P+P_{0}(\Delta A)\right), \quad \Delta A \in \mathscr{U},
$$

where $P_{A A}$ satisfies (7), and

$$
\mathscr{T}(\mathscr{U}) \leq \frac{1}{1-\gamma}\left[\operatorname{tr}(P V)+\sup _{\Delta A \in \mathscr{Z}} \operatorname{tr}\left(P_{0}(\Delta A) V\right)\right]
$$


In addition, if there exists $\bar{P}_{0} \in \mathscr{S}^{n}$ such that

$$
P_{0}(\Delta A) \leq \bar{P}_{0},
$$

then

$$
\mathscr{T}(\mathscr{U}) \leq \frac{1}{1-\gamma} \operatorname{tr}\left[\left(P+\bar{P}_{0}\right) V\right] .
$$

Proof. Note that, for all $\Delta A \in \mathscr{U},(8)$ is equivalent to

$$
\begin{aligned}
0= & (A+\Delta A)^{\mathrm{T}}\left(P+P_{0}(\Delta A)\right)+\left(P+P_{0}(\Delta A)\right)(A+\Delta A)+\Omega_{0}(P)+R \\
& -\left[(A+\Delta A)^{\mathrm{T}} P_{0}(\Delta A)+P_{0}(A A)(A+\Delta A)\right]-\left(\Lambda A^{\mathrm{T}} P+P A A\right) \\
= & (A+\Delta A)^{\mathrm{T}}\left(P+P_{0}(\Delta A)\right)+\left(P+P_{0}(\Delta A)\right)(A+\Delta A)+R-R_{0}+R_{0}^{\prime},
\end{aligned}
$$

where

$$
\begin{aligned}
R_{0}^{\prime} & \triangleq \Omega_{0}(P)+R_{0}-\left[(A+\Delta A)^{\mathrm{T}} P_{0}(\Delta A)+P_{0}(\Delta A)(A+\Delta A)\right]-\left(\Delta A^{\mathrm{T}} P+P \Delta A\right) \\
& =\Omega(P, A A)+R_{0}-\left(A A^{\mathrm{T}} P+P A A\right) .
\end{aligned}
$$

Hence, (18) has a solution $P \in \mathscr{A}^{n}$ for all $\triangle A \in \mathscr{U}$. Thus, if the detectability condition (12) holds for all $\Delta A \in \mathscr{U}$, then it follows from [21, Theorem 3.6] that $\left(R-R_{0}+R_{0}^{\prime}, A+\Delta A\right)$ is detectable, $\triangle A \in \mathscr{U}$. It now follows from (18) and [21, Lemma 12.2] that $A+\Delta A$ is asymptotically stable, $\Delta A \in \mathscr{U}$. Conversely, if $A+\Delta A$ is asymptotically stable for all $\Delta A \in \mathscr{U}$, then (12) is immediate.

Now, subtracting $(1-\gamma) \cdot(7)$ from $(18)$ yields

$$
\begin{aligned}
0= & (A+\Delta A)^{\mathrm{T}}\left(P+P_{0}(\Delta A)-(1-\gamma) P_{\Delta A}\right)+\left(P+P_{0}(\Delta A)-(1-\gamma) P_{\Delta A}\right)(A+\Delta A) \\
& +R_{0}^{\prime}-R_{0}+\gamma R, \Delta A \in \mathscr{U},
\end{aligned}
$$

or, since $A+\Delta A$ is asymptotically stable for all $\Delta A \in \mathscr{U}$ and $R_{0} \leq \gamma R,(19)$ implies that, for all $\Delta A \in \mathscr{U}$,

$$
\begin{aligned}
P+P_{0}(\Delta A)-(1-\gamma) P_{\Delta A} & =\int_{0}^{\infty} \mathrm{e}^{(A \cdot \Delta A)^{\mathrm{T}} \mathrm{t}}\left[R_{0}^{\prime}+\gamma R-R_{0}\right] \mathrm{e}^{(A+\Delta A) t} \mathrm{~d} t \\
& \geq \int_{0}^{\infty} \mathrm{e}^{(A-\Delta A)^{\prime} t} R_{0}^{\prime} \mathrm{e}^{(A+\Delta A) r} \mathrm{~d} t \\
& \geq 0
\end{aligned}
$$

which implies (14).

Next, using (14), it follows from (5) that

$$
\begin{aligned}
& \mathscr{T}(\mathscr{U})=\sup _{A A \in \mathscr{H}} \operatorname{tr}\left(D^{\mathrm{T}} P_{\Delta A} D\right) \leq \frac{1}{1-\gamma_{\Delta A \in \mathscr{H}}} \sup _{\Delta,} \operatorname{tr}\left[D^{\mathrm{T}}\left(P+P_{0}(\Delta A)\right) D\right] \\
& =\frac{1}{1-\gamma}\left[\operatorname{tr}(P V)+\sup _{\Delta A E^{\prime \prime}} \operatorname{tr}\left(P_{0}(\Delta A) V\right)\right] \text {, }
\end{aligned}
$$

which yields (15). Furthermore, using (16) it follows that

$$
\begin{aligned}
\mathscr{T}(\mathscr{U}) \leq \frac{1}{1-\gamma}\left[\operatorname{tr}(P V)+\sup _{\Delta A \in \mathscr{U}} \operatorname{tr}\left(P_{0}(\Delta A) V\right)\right] & \leq \frac{1}{1-\gamma}\left[\operatorname{tr}(P V)+\operatorname{tr}\left(\bar{P}_{0} V\right)\right] \\
& =\frac{1}{1-\gamma} \operatorname{tr}\left[\left(P+\bar{P}_{0}\right) V\right] .
\end{aligned}
$$


Remark 2.3. Theorem 2.2 is a generalization of Theorem 3.1 of [15]. Specifically, the bound in [15] is required to hold for all nonnegative-definite matrices, whereas in Theorem 2.2 equation (9) need only hold for the solution $P$ of (8). Furthermore, in [15], $R_{0}=0$.

Remark 2.4. Inequality (9) is equivalent to

$$
(A+\Delta A)^{\mathrm{T}}\left(P+P_{0}(\Delta A)\right)+\left(P+P_{0}(\Delta A)\right)(A+\Delta A)+R-R_{0} \leq 0,
$$

which shows that $V(x)=x^{\mathrm{T}}\left(P+P_{0}(\Delta A)\right) x$ is a Lyapunov function corresponding to $A+\Delta A$. In constructing this Lyapunov function, the matrix $P$ can be viewed as a predictor term, $P_{0}(\Delta A)$ provides a corrector term, and $P_{\mathrm{T}} \triangleq P+P_{0}(A A)$ is the total Lyapunov matrix.

Remark 2.5. If $P_{0}(\Delta A)$ is independent of $\Delta A$, then by choosing $\bar{P}_{0}=P_{0}(\Delta A)$ it follows that (15) is identical to (17).

\section{Application to the maximum-entropy covariance model}

Now we specialize to the case in which $\mathscr{U}$ is given by

$$
\mathscr{U} \triangleq\left\{\Delta A \in \mathbb{R}^{n \times n}: \Delta A=\sum_{i=1}^{r} \sigma_{i} A_{i},\left|\sigma_{i}\right| \leq \delta_{i}, \quad i=1, \ldots, r\right\},
$$

where $\delta_{i}>0$ and the matrices $A_{i} \in \mathbb{R}^{n \times n}$, which represent the uncertainty structure, are the given skewsymmetric matrices, that is, $A_{i}+A_{i}^{\mathrm{T}}=0, i=1, \ldots, r$. In addition, we assume that $A+A^{\mathrm{T}}<0$. This formulation can be viewed as the representation of a dissipative system (such as a flexible structure) with energy-conserving perturbations. This property can be seen by means of the Lyapunov function $V(x)=x^{\mathrm{T}} x$ whose decay rate is independent of $\sigma_{i}$. Thus, $A+\Delta A$ is uniformly asymptotically stable even for arbitrarily time-varying $\sigma_{i}(t)$. For simplicity, however, we confine our analysis to constant parameter uncertainty. In addition, although the system is robustly stable for time-varying parameter uncertainties, the performance bounds we obtain via Theorem 2.2 are valid only for the case of constant parameter uncertainty.

We now introduce a specific choice of $\Omega_{0}(P)$ that is motivated by the maximum-entropy covariance model. Specifically, as in $[18]$ we choose

$$
\Omega_{0}(P)=\sum_{i=1}^{r} \delta_{i}^{2}\left(\frac{1}{2} A_{i}^{2 \mathrm{~T}} P+A_{i}^{\mathrm{T}} P A_{i}+\frac{1}{2} P A_{i}^{2}\right) .
$$

First we prove that with this choice of $\Omega_{0}(P)$ equation (8) has a unique solution. Then we show that, when $r=1$, equation (8) has an asymptotic solution for $\delta_{1} \rightarrow \infty$.

Proposition 3.1. Assume that $A+A^{\mathrm{T}}<0, A_{i}+A_{i}^{\mathrm{T}}=0$, and $\delta_{i} \geq 0, i=1, \ldots, r$. Then there exists a unique matrix $P \in \mathbb{R}^{n \times n}$ satisfying

$$
0=A^{\mathrm{T}} P+P A+\sum_{i=1}^{r} \delta_{i}^{2}\left(\frac{1}{2} A_{i}^{2 \mathrm{~T}} P+A_{i}^{\mathrm{T}} P A_{i}+\frac{1}{2} P A_{i}^{2}\right)+R .
$$

Furthermore, $P$ is nonnegative-definite.

Proof. Applying the "vec" operator [7] to (22) yields

$$
0=\mathscr{A}^{\mathrm{T}} \operatorname{vec} P+\operatorname{vec} R,
$$

where

$$
\mathscr{A} \triangleq(A \oplus A)+\sum_{i=1}^{r} \frac{1}{2} \delta_{i}^{2}\left(A_{i} \oplus A_{i}\right)^{2}
$$


and $\oplus$ and later $\otimes$ denote Kronecker sum and product, respectively. Since $A+A^{\mathrm{T}}<0$, it follows that $(A \oplus A)+(A \oplus A)^{\mathrm{T}}=\left(A+A^{\mathrm{T}}\right) \oplus\left(A+A^{\mathrm{T}}\right)<0$. In addition, the assumption that $A_{i}$ is skew-symmetric implies that $A_{i} \oplus A_{i}$ is also skew-symmetric and thus $\left(A_{i} \oplus A_{i}\right)^{2} \leq 0, i=1, \ldots, r$. Thus, $\mathscr{A}^{\mathrm{T}}<0$, which implies that $\mathscr{A}$ is asymptotically stable. Thus, (23) yields $P=\operatorname{vec}^{-1}\left(-\mathscr{A}^{-\mathrm{T}}\right.$ vec $\left.R\right)$. This proves existence and uniqueness.

Next, we show that $P$ is nonnegative-definite. Note that since $-\mathscr{A}^{-\mathrm{T}}=\int_{0}^{\infty} \mathrm{e}^{\alpha^{\mathrm{T}} t} \mathrm{~d} t$, we can write

$$
P=\operatorname{vec}^{-1}\left(\int_{0}^{\infty} \mathrm{e}^{f^{\mathrm{T}_{t}}} \operatorname{vec} R \mathrm{~d} t\right)
$$

After some manipulation (24) can be written as

$$
P=\operatorname{vec}^{-1}\left(\int_{0}^{x_{i}} \exp \left(t\left[\sum_{i=1}^{r}\left(\frac{A}{r}+\frac{1}{2} \delta_{i}^{2} A_{i}^{2}\right)^{\mathrm{T}} \oplus \sum_{i=1}^{r}\left(\frac{A}{r}+\frac{1}{2} \delta_{i}^{2} A_{i}^{2}\right)^{\mathrm{T}}+\sum_{i=1}^{r} \frac{1}{2} \delta_{i}^{2}\left(A_{i} \otimes A_{i}\right)^{\mathrm{T}}\right]\right) \operatorname{vec} R \mathrm{~d} t\right) .
$$

Now, using the exponential product formula it follows that

$$
\begin{aligned}
P=\operatorname{vec}^{-1}\left(\int_{0}^{\infty} \lim _{m \rightarrow \infty}[\right. & \left.\exp \left(\frac{t}{n}\left[\sum_{i=1}^{r}\left(\frac{A^{\mathrm{T}}}{r}+\frac{1}{2} \delta_{i}^{2} A_{i}^{2 \mathrm{~T}}\right) \oplus \sum_{i=1}^{r}\left(\frac{A^{\mathrm{T}}}{r}+\frac{1}{2} \delta_{i}^{2} A_{i}^{2 \mathrm{~T}}\right)\right)\right]\right) \\
& \left.\times \prod_{i=1}^{r} \exp \left(\frac{\delta_{i}^{2} t}{2 m}\left(A_{i}^{\mathrm{T}} \otimes A_{i}^{\mathrm{T}}\right)\right]^{m} \operatorname{vec} R \mathrm{~d} t\right) .
\end{aligned}
$$

For simplicity, we assume $r=1$. If $r>1$ only minor modifications are needed. First fix $m$ and let $R_{(0)} \triangleq R_{\text {; }}$ define the series $Z_{(j)}, R_{(j)}, j=0,1, \ldots, m-1$, by

$$
\begin{aligned}
\operatorname{vec} Z_{(j+1)}(t) & \triangleq \mathrm{e}^{\left(\delta_{1}^{2} t i 2 m\right)\left(A_{1} \otimes A_{1} !^{\mathrm{T}}\right.} \operatorname{vec} R_{(j)}(t)=\operatorname{vec} \sum_{k=0}^{\infty} \frac{1}{k !}\left(\frac{\delta_{1}^{2} t}{2 m}\right)^{k} A_{1}^{k \mathrm{~T}} R_{(j)}(t) A_{1}^{k}, \\
\operatorname{vec} R_{(j+1)}(t) & \triangleq \exp \left(\begin{array}{c}
t \\
m
\end{array}\left(\begin{array}{c}
A+\delta_{1}^{2} \\
2
\end{array} A_{1}^{2}\right) \oplus\left(A+\frac{\delta_{1}^{2}}{2} A_{1}^{2}\right)^{\mathrm{T}}\right) \operatorname{vec} Z_{(j+1)}(t) \\
& =\operatorname{vec} \exp \left(\begin{array}{c}
t \\
m
\end{array}\left(A+\frac{\delta_{1}^{2}}{2} A_{1}^{2}\right)^{\mathrm{T}}\right) Z_{(j+1)}(t) \exp \left(\frac{t}{m}\left(A+\frac{\delta_{1}^{2}}{2} A_{1}^{2}\right)\right)
\end{aligned}
$$

It is obvious that both $Z_{(j)}(t)$ and $R_{(j)}(t)$ are nonnegative-definite matrices for all $j=0,1, \ldots, m-1$ and $t \geq 0$. Finally, since $m$ is arbitrary, it can be shown that

$$
P=\operatorname{vec}^{-1}\left(\int_{0}^{\infty} \lim _{m \rightarrow \infty} \operatorname{vec} R_{(m)} \mathrm{d} t\right)=\int_{0}^{\infty} \lim _{m \rightarrow \infty} R_{(m)} \mathrm{d} t \geq 0 .
$$

Next we show that (22) with $r=1$ has an asymptotic solution for $\delta_{1} \rightarrow \infty$. First, we need the following definition and lemma.

Definition 3.2. For $F \in \mathbb{R}^{n \times n}$, the smallest nonnegative integer $k$ such that $\operatorname{rank}\left(F^{k}\right)=\operatorname{rank}\left(F^{k+1}\right)$ is called the index of $F$ and is denoted by Ind $(F)[8]$.

Remark 3.3. If $F$ is invertible, Ind $(F)=0$. Also Ind $(0)=1$. We adopt the convention that $0^{\circ}=1[8]$.

Definition 3.4. A matrix $F \in \mathbb{R}^{n \times n}$ is called EP [8] if either $F$ is invertible or there exists an orthogonal matrix $U \in \mathbb{R}^{n \times n}$ and an invertible matrix $F_{1} \in \mathbb{R}^{m \times m}$, where $m \leq n$, such that

$$
F=U\left[\begin{array}{cc}
F_{1} & 0 \\
0 & 0
\end{array}\right] U^{\mathrm{T}}
$$


Remark 3.5. If $F$ is EP, then Ind $(F) \leq 1$, and the group inverse $F^{\#}$ of $F$ is given by [8]

$$
F^{\#}=U\left[\begin{array}{cc}
F_{1}^{-1} & 0 \\
0 & 0
\end{array}\right] U^{\mathrm{T}} \text {. }
$$

Lemma 3.6. Let $A, B \in \mathbb{R}^{n \times n}$, where $A+A^{\mathrm{T}}<0$ and $B$ is an EP matrix. Then

$$
\text { Ind }(A B)=\operatorname{Ind}(B) \text {. }
$$

Proof. Since $B$ is an EP matrix, Remark 3.5 implies that Ind $(B) \leq 1$. Hence, we consider two cases.

(1) Suppose Ind $(B)=0$, so that $B$ is invertible. Since $A+A^{\mathrm{T}}<0$, it follows that $A$ is asymptotically stable and hence invertible. Therefore, $A B$ is invertible and thus $\operatorname{Ind}(A B)=0$.

(2) Suppose Ind $(B)=1$, and let $\operatorname{rank}(B)=n-r$, where $r \geq 1$. Since $B$ is an EP matrix, there exists an orthogonal matrix $U$ and a matrix $D_{B}$ such that $B=U D_{B} U^{\mathrm{T}}$, where

$$
D_{B}=\left[\begin{array}{rr}
B_{1} & 0 \\
0 & 0
\end{array}\right], \quad B_{1} \in \mathbb{R}^{(n-r) \times(n-r)}, \operatorname{det}\left(B_{1}\right) \neq 0 .
$$

Since rank $(A B)=n-r$, it suffices to show that the zero eigenvalue of $A B$ has multiplicity $r$.

By writing $U^{\mathrm{T}} A U$ in the form

$$
A^{\prime} \triangleq U^{T} A U=\left[\begin{array}{ll}
A_{11}^{\prime} & A_{12}^{\prime} \\
A_{21}^{\prime} & A_{22}^{\prime}
\end{array}\right]
$$

where $A_{11}^{\prime} \in \mathbb{R}^{(n-r) \times(n-r)}, A_{22}^{\prime} \in \mathbb{R}^{r \times r}, A_{12}^{\prime} \in \mathbb{R}^{(n-r) \times r}, A_{21}^{\prime} \in \mathbb{R}^{r \times(n-r)}$, we have

$$
U^{\mathrm{T}} A U D_{B}=\left[\begin{array}{lll}
A_{11}^{\prime} B_{1} & 0 \\
A_{21}^{\prime} B_{1} & 0
\end{array}\right] \text {. }
$$

Consequently, the characteristic polynomial of $A B$ is

$$
\begin{aligned}
\operatorname{det}(\lambda I-A B) & =\operatorname{det}\left(\lambda I-U\left(U^{\mathrm{T}} A U D_{B}\right) U^{\mathrm{T}}\right)=\operatorname{det}\left(\lambda I-U^{\mathrm{T}} A U D_{B}\right) \\
& =\operatorname{det}\left[\begin{array}{cc}
\lambda I_{n-r}-A_{11}^{\prime} B_{1} & 0 \\
-A_{21}^{\prime} B_{1} & \lambda I_{r}
\end{array}\right]=\lambda^{r} \operatorname{det}\left(\lambda I_{n-r}-A_{11}^{\prime} B_{1}\right) .
\end{aligned}
$$

Equation (28) implies that the zero eigenvalue of $A B$ has at least multiplicity $r$.

The final step is to show that $A_{11}^{\prime} B_{1}$ has no zero eigenvalue or, equivalently, $\operatorname{det}\left(A_{11}^{\prime} B_{1}\right) \neq 0$. Since $A+A^{\mathrm{T}}<0$, it follows that $U^{\mathrm{T}}\left(A+A^{\mathrm{T}}\right) U<0$, that is, $A^{\prime}+A^{\prime \mathrm{T}}<0$. Thus, $A_{11}^{\prime}+\left(A_{11}^{\prime}\right)^{\mathrm{T}}<0$, which implies that $A_{11}^{\prime}$ is asymptotically stable. Therefore, we have $\operatorname{det}\left(A_{11}^{\prime}\right) \neq 0$. Noting

$$
\operatorname{det}\left(A_{11}^{\prime} B_{1}\right)=\operatorname{det}\left(A_{11}^{\prime}\right) \operatorname{det}\left(B_{1}\right) \neq 0
$$

completes the proof.

For convenience, we define

$$
A \triangleq\left(A^{\mathrm{T}} \oplus A^{\mathrm{T}}\right)^{-1}\left(A_{1}^{\mathrm{T}} \oplus A_{1}^{\mathrm{T}}\right)^{2} .
$$

Lemma 3.7. Let $A, A_{1} \in \mathbb{R}^{n \times n}$, where $A+A^{\mathrm{T}}<0$ and $A_{1}+A_{1}^{\mathrm{T}}=0$. Then $\operatorname{Ind}(\Lambda)=1$.

Proof. Since $A_{1}$ is skew-symmetric, it follows that $A_{1} \oplus A_{1}$ is also skew-symmetric. Thus, $\left(A_{1} \oplus A_{1}\right)^{2}$ is symmetric (actually, it is negative-semidefinite) and hence is EP. In addition, it is obvious that $A_{1} \oplus A_{1}$ is singular. Thus, Ind $\left(A_{1}^{\mathrm{T}} \oplus A_{1}^{\mathrm{T}}\right)^{2}=1$. Furthermore, since $A+A^{\mathrm{T}}<0$ implies $(A \oplus A)+\left(A^{\mathrm{T}} \oplus A^{\mathrm{T}}\right)<0$ and equivalently implies $(A \oplus A)^{-1}+\left(A^{\mathrm{T}} \oplus A^{\mathrm{T}}\right)^{-1}<0$, it follows from Lemma 3.6 that Ind $(\Lambda)=1$. 
We are now ready to prove the existence of an asymptotic solution of equation (8) when $r=1$. For notational convenience, we replace $\delta_{1}^{2} / 2$ by $\alpha$.

Proposition 3.8. Let $A, A_{1} \in \mathbb{R}^{n \times n}, R \in \mathcal{A}^{n}$ and $\alpha \geq 0$. Furthermore, assume that $A+A^{\mathrm{T}}<0, A_{1}+A_{1}^{\mathrm{T}}=0$, and let $P_{\alpha} \in \mathcal{A}^{n}$ be the unique, nonnegative-definite solution to

$$
0=A^{\mathrm{T} P}+P A+\alpha\left(A_{1}^{2 \mathrm{~T}} P+2 A_{1}^{\mathrm{T}} P A_{1}+P A_{1}^{2}\right)+R .
$$

Then $P_{\infty} \triangleq \lim _{x \rightarrow x} P_{\alpha}$ exists and is given by

$$
P_{x}=\operatorname{vec}^{-1}\left[\left(I-A A^{*}\right)\left(A^{\mathrm{T}} \oplus A^{\mathrm{T}}\right)^{-1}(-\operatorname{vec} R)\right] .
$$

Proof. Applying the vec operator to equation (30) yields

$$
0=\left[\left(A^{\mathrm{T}} \oplus A^{\mathrm{T}}\right)+\alpha\left(A_{1}^{\mathrm{T}} \oplus A_{1}^{\mathrm{T}}\right)^{2}\right] \operatorname{vec} P+\operatorname{vec} R,
$$

so that

$$
\operatorname{vec} P=[I+\alpha A]^{-1}\left(A^{\mathrm{T}} \oplus A^{\mathrm{T}}\right)^{-1}(-\operatorname{vec} R),
$$

and we can write $P_{\infty}$ as

$$
\begin{aligned}
\operatorname{vec} P_{\infty} & =\lim _{\alpha \rightarrow \infty}(I+\alpha A)^{-1}\left(A^{\mathrm{T}} \oplus A^{\mathrm{T}}\right)^{-1}(-\operatorname{vec} R) \\
& =\lim _{\alpha \rightarrow \infty}\left[\alpha\left(\frac{1}{\alpha} I+A\right)\right]^{-1}\left(A^{\mathrm{T}} \oplus A^{\mathrm{T}}\right)^{-1}(-\operatorname{vec} R) \\
& =\lim _{z \rightarrow \infty} z(z I+\Lambda)^{-1}\left(A^{\mathrm{T}} \oplus A^{\mathrm{T}}\right)^{-1}(-\operatorname{vec} R) .
\end{aligned}
$$

Now since Ind $(A)=1$, it follows from [8, Theorem 7.6.2] that the above limit exists and is given by vec $P_{x}=\left(I-\Lambda A^{*}\right)\left(A^{\mathrm{T}} \oplus A^{\mathrm{T}}\right)^{-1}(-\operatorname{vec} R)$, which yields (31).

For the following result, define the commutator $[F, G] \triangleq F G-G F$.

Lemma 3.9. Let $A, A_{1} \in \mathbb{R}^{n \times n}, R \in \mathscr{N}^{n}$. Furthermore, suppose that $A+A^{\mathrm{T}}<0, A_{1}+A_{1}^{\mathrm{T}}=0$, and let $P_{\infty} \in \mathscr{N}^{n}$ be given by (31). Then $P_{x}$ satisfies

$$
\left[A_{1}^{\mathrm{T}}, P_{\propto}\right]=0 .
$$

Proof. Since $A_{1}$ is skew-symmetric, we have

$$
\begin{aligned}
\operatorname{vec}\left[A_{1}^{\mathrm{T}}, P_{\infty}\right]=\operatorname{vec}\left(A_{1}^{\mathrm{T}} P_{\infty}+P_{\infty} A_{1}\right) & =\left(A_{1}^{\mathrm{T}} \oplus A_{1}^{\mathrm{T}}\right) \operatorname{vec} P_{\infty} \\
& =\left(A_{1}^{\mathrm{T}} \oplus A_{1}^{\mathrm{T}}\right)\left(I-\Lambda A^{\#}\right)\left(A^{\mathrm{T}} \oplus A^{\mathrm{T}}\right)^{-1}(-\operatorname{vec} R),
\end{aligned}
$$

where $A$ is defined by (29). Since, by Lemma 3.7, Ind $(A)=1$, it follows from Remark 3.5 that $A$ and $A^{*}$ can be expressed in the form

$$
A=V\left[\begin{array}{ll}
C & 0 \\
0 & 0
\end{array}\right] V^{-1}, \quad A^{*}=V\left[\begin{array}{cc}
C^{-1} & 0 \\
0 & 0
\end{array}\right] V^{-1},
$$

where $\operatorname{det}(C) \neq 0$. Writing $V=\left[V_{1} V_{2}\right]$, the identity

$$
\Lambda V=V\left[\begin{array}{ll}
C & 0 \\
0 & 0
\end{array}\right]
$$


implies that $A V_{2}=0$. Consequently, $\left(A_{1}^{\mathrm{T}} \oplus A_{1}^{\mathrm{T}}\right)^{2} V_{2}=0$, and, since $\operatorname{Ind}\left(A_{1}^{\mathrm{T}} \oplus A_{1}^{\mathrm{T}}\right)=1$, it follows that $\left(A_{1}^{\mathrm{T}} \oplus A_{1}^{\mathrm{T}}\right) V_{2}=0$. Therefore, equation (33) can be written as

$$
\begin{aligned}
\operatorname{vec}\left[A_{1}^{\mathrm{T}}, P_{\infty}\right] & =\left(A_{1}^{\mathrm{T} \oplus} A_{1}^{\mathrm{T}}\right)\left(I-V\left[\begin{array}{ll}
I & 0 \\
0 & 0
\end{array}\right] V^{-1}\right)\left(A^{\mathrm{T}} \oplus A^{\mathrm{T}}\right)^{-1}(-\operatorname{vec} R) \\
& =\left(A_{1}^{\mathrm{T}} \oplus A_{1}^{\mathrm{T}}\right)\left(V\left[\begin{array}{ll}
0 & 0 \\
0 & I
\end{array}\right] V^{-1}\right)\left(A^{\mathrm{T}} \oplus A^{\mathrm{T}}\right)^{-1}(-\operatorname{vec} R) \\
& =\left(A_{1}^{\mathrm{T}} \oplus A_{1}^{\mathrm{T}}\right)\left[0 V_{2}\right] V^{-1}\left(A^{\mathrm{T}} \oplus A^{\mathrm{T}}\right)^{-1}(-\operatorname{vec} R) \\
& =\left[0\left(A_{1}^{\mathrm{T}} \oplus A_{1}^{\mathrm{T}}\right) V_{2}\right] V^{-1}\left(A^{\mathrm{T}} \oplus A^{\mathrm{T}}\right)^{-1}(-\operatorname{vec} R)=0 .
\end{aligned}
$$

As a result, $\left[A_{1}^{\mathrm{T}}, P_{\infty}\right]=0$.

Remark 3.10. If $P$ is symmetric, $A_{1}$ is skew-symmetric, then it can be shown that $\left[A_{1}^{\mathrm{T}},\left[A_{1}^{\mathrm{T}}, P_{\infty}\right]\right]=0$ if and only if $\left[A_{1}^{\mathrm{T}}, P_{\infty}\right]=0$. This fact is of interest since (21) can be written as

$$
\Omega_{0}(P)=\sum_{i=1}^{r} \frac{1}{2} \delta_{i}^{2}\left[A_{i}^{\mathrm{T}},\left[A_{i}^{\mathrm{T}}, P\right]\right] .
$$

Thus, if $r=1$ and $\delta_{1} \rightarrow \infty$, then $\left[A_{1}^{\mathrm{T}},\left[A_{1}^{\mathrm{T}}, P_{\infty}\right]\right] \rightarrow 0$. Note $\left(\delta_{1}^{2} / 2\right)\left[A_{1}^{\mathrm{T}},\left[A_{1}^{\mathrm{T}}, P_{\infty}\right]\right]=-\left(A^{\mathrm{T}} P_{\infty}+\right.$ $\left.P_{\infty} A+R\right)=-\operatorname{vec}^{-1}\left[\left(A_{1}^{\mathrm{T}} \oplus A_{1}^{\mathrm{T}}\right)^{2}\left[\left(A^{\mathrm{T}} \oplus A^{\mathrm{T}}\right)^{-1}\left(A_{1}^{\mathrm{T}} \oplus A_{1}^{\mathrm{T}}\right)^{2}\right]^{*}\left(A^{\mathrm{T}} \oplus A^{\mathrm{T}}\right)^{-1} \operatorname{vec} R\right]$.

\section{The choice of corrector term $P_{0}$}

Now we propose a corrector term $P_{0}$ for the case of general skew-symmetric matrices $A_{i} \in \mathbb{R}^{n \times n}, i=1, \ldots, r$, where $r \geq 1$. For a symmetric matrix $B$, define $|B| \triangleq \sqrt{B^{2}}$.

Proposition 4.1. Assume $A+A^{\mathrm{T}}<0, A_{i}+A_{i}^{\mathrm{T}}=0$, and $\delta_{i} \geq 0, i=1, \ldots, r$. Let $P \in \mathscr{N}^{n}$ satisfy (22) and let

$$
\beta \geq \max \left\{\sum_{i=1}^{r} \mu_{i},-\lambda_{\min }(P)\right\},
$$

where, for $i=1, \ldots, r$,

$$
\mu_{\mathrm{i}} \triangleq \lambda_{\max }\left(\left(\delta_{i}\left|\left[A_{i}^{\mathrm{T}}, P\right]\right|-\frac{1}{2} \delta_{i}^{2}\left[A_{i}^{\mathrm{T}},\left[A_{i}^{\mathrm{T}}, P\right]\right]\right)\left(-A-A^{\mathrm{T}}\right)^{-1}\right) .
$$

If $P_{0}(\Delta A) \triangleq \beta I_{n}$, then (9) and (10) are satisfied with $R_{0}=0$ and $\mathscr{U}$ given by (20).

Proof. By substituting $P_{0}(\Delta A)=B I_{n}$ into (9) with $R_{0}=0$ and letting $G=\sqrt{-A^{\mathrm{T}}-A}$, we have

$$
\begin{aligned}
& \Omega(P, \Delta A)+R_{0}-\left(A A^{\mathrm{T}} P+P \Delta A\right) \\
& \quad=\beta\left(-A^{\mathrm{T}}-A\right)-\sum_{i=1}^{r} \sigma_{i}\left[A_{i}^{\mathrm{T}}, P\right]+\sum_{i=1}^{r} \frac{1}{2} \delta_{i}^{2}\left[A_{i}^{\mathrm{T}},\left[A_{i}^{\mathrm{T}}, P\right]\right] \\
& \quad \geq \beta\left(-A^{\mathrm{T}}-A\right)-\sum_{i=1}^{r} \delta_{i}\left|\left[A_{i}^{\mathrm{T}}, P\right]\right|+\sum_{i=1}^{r} \frac{1}{2} \delta_{i}^{2}\left[A_{i}^{\mathrm{T}},\left[A_{i}^{\mathrm{T}}, P\right]\right] \\
& \quad=G\left\{\beta I_{n}-\sum_{i=1}^{r} G^{-1}\left(\delta_{i}\left|\left[A_{i}^{\mathrm{T}}, P\right]\right|-\frac{1}{2} \delta_{i}^{2}\left[A_{i}^{\mathrm{T}},\left[A_{i}^{\mathrm{T}}, P\right]\right]\right) G^{-1}\right\} G \\
& \quad \geq G\left\{\beta I_{n}-\sum_{i=1}^{r} \lambda_{\max }\left(G^{-1}\left(\delta_{i}\left|\left[A_{i}^{\mathrm{T}}, P\right]\right|-\frac{1}{2} \delta_{i}^{2}\left[A_{i}^{\mathrm{T}},\left[A_{i}^{\mathrm{T}}, P\right]\right]\right) G^{-1}\right) I_{n}\right\} G
\end{aligned}
$$




$$
\begin{aligned}
& =G\left\{\beta I_{n}-\sum_{i=1}^{r} \lambda_{\max }\left(\left(\delta_{i}\left|\left[A_{i}^{\mathrm{T}}, P\right]\right|-\frac{1}{2} \delta_{i}^{2}\left[A_{i}^{\mathrm{T}},\left[A_{i}^{\mathrm{T}}, P\right]\right]\right)\left(-A-A^{\mathrm{T}}\right)^{-1}\right) I_{n}\right\} G \\
& =G\left\{\beta I_{n}-\sum_{i=1}^{r} \mu_{i} I_{n}\right\} G \\
& \geq 0
\end{aligned}
$$

which proves (9). Finally, it is obvious that $P+P_{0}(A A)=P+\beta I_{n} \geq \lambda_{\min }(P) I_{n}+\beta I_{n} \geq 0$, so that (10) is satisfied.

Henceforth, we confine our attention to the special case $r=1$ and

$$
A=\left[\begin{array}{cc}
-\eta & \omega \\
-\omega & -\eta
\end{array}\right], \quad A_{1}=\left[\begin{array}{rc}
0 & 1 \\
-1 & 0
\end{array}\right],
$$

where $\eta>0$ and $\omega \in \mathbb{R}$. For notational convenience, we adopt the traditional symbol $J$ for $A_{1}$. In this case $\Omega_{0}(P)$ given by $(21)$ has the form

$$
\Omega_{0}(P)=\delta_{1}^{2}\left(\frac{1}{2} J^{2 \mathrm{~T}} P+J^{\mathrm{T}} P J+\frac{1}{2} P J^{2}\right) .
$$

Note that $J^{\mathrm{T}}=-J$ and $J^{2}=-I_{2}$, where $I_{2}$ denotes the $2 \times 2$ identity matrix.

Proposition 4.2. Assume that $R$ is positive-definite and let $P$ satisfy

$$
0=A^{\mathrm{T}} P+P A+\delta_{1}^{2}\left(\frac{1}{2} J^{2 \mathrm{~T}} P+J^{\mathrm{T}} P J+\frac{1}{2} P J^{2}\right)+R,
$$

let $\gamma<1$, and define

$$
P_{0}(\Delta A) \triangleq(1-\gamma) J^{\mathrm{T}} P J-\gamma P, \quad \Delta A \in \mathscr{U} .
$$

Then (9) and (10) are satisfied with $R_{0}=\gamma R$. Furthermore, the performance bound (15) is given by

$$
\mathscr{T}(\mathscr{Z}) \leq \operatorname{tr}(V) \operatorname{tr}(P) .
$$

Proof. Clearly, (10) is satisfied. Secondly, since

$$
A J=J A, \quad J J^{\mathrm{T}}=J^{\mathrm{T}} J=I_{2}, \quad J^{\mathrm{T}} \Omega_{0}(P) J=-\Omega_{0}(P),
$$

and $P$ satisfies (37), it follows that

$$
\begin{aligned}
\Omega_{0}(P)+R_{0}-\left[\left(A+\sigma_{1} J\right)^{\mathrm{T}} P_{0}+P_{0}\left(A+\sigma_{1} J\right)\right]-\sigma_{1}\left(J^{\mathrm{T}} P+P J\right) \\
=\Omega_{0}(P)+R_{0}-\left[(1-\gamma)\left(A^{\mathrm{T}} J^{\mathrm{T}} P J+J^{\mathrm{T}} P J A\right)+\sigma_{1}(1-\gamma)\left(J^{\mathrm{T}} J^{\mathrm{T}} P J+J^{\mathrm{T}} P J J\right)\right. \\
\left.\quad-\gamma\left(A^{\mathrm{T}} P+P A\right)-\sigma_{1} \gamma\left(J^{\mathrm{T}} P+P J\right)\right]-\sigma_{1}\left(J^{\mathrm{T}} P+P J\right) \\
=\Omega_{0}(P)+R_{0}-(1-\gamma) J^{\mathrm{T}}\left(A^{\mathrm{T}} P+P A\right) J+\gamma\left(A^{\mathrm{T}} P+P A\right) \\
=\Omega_{0}(P)+R_{0}-(1-\gamma) J^{\mathrm{T}}\left(-\Omega_{0}(P)-R\right) J+\gamma\left(-\Omega_{0}(P)-R\right) \\
=R_{0}-\gamma R+(1-\gamma) J^{\mathrm{T}} R J \\
\geq 0 .
\end{aligned}
$$

Finally, we have

$$
\begin{aligned}
\mathscr{T}(\mathscr{U}) \leq \frac{1}{1-\gamma}\left[\operatorname{tr}(P V)+\operatorname{tr}\left(P_{0} V\right)\right] & =\operatorname{tr}(P V)+\operatorname{tr}\left(J^{\mathrm{T}} P J V\right) \\
& =\operatorname{tr}\left[P\left(V+J V J^{\mathrm{T}}\right)\right]=\operatorname{tr}(V) \operatorname{tr}(P) .
\end{aligned}
$$


Remark 4.3. Note that unlike the parameter-dependent Lyapunov function used in [15] for the Popov criterion, the auxiliary portion $P_{0}(\Delta A)$ given by (38) is independent of $\sigma_{1}$. Therefore, this auxiliary portion $P_{0}(\Delta A)$ guarantees robust stability with respect to time-varying $\sigma_{1}(t)$. This robust stability property was already shown at the beginning of this section by means of the Lyapunov function $V(x)=x^{\mathrm{T}} x$.

Remark 4.4. Since by Proposition 3.1, equation (37) has a solution for all $\delta_{1}>0$, it follows that robust stability is guaranteed for arbitrary $\sigma_{1}$, that is, not necessarily bounded by $\delta_{1}$.

Remark 4.5. It is easy to show that $\operatorname{tr}(P)=(1 / 2 \eta) \operatorname{tr}(R)$ and $P_{T}=P+P_{0}=(1-\gamma)\left(J^{\mathrm{T}} P J+P\right)$ $=(1-\gamma) \operatorname{tr}(P) I_{2}$. Thus, (39) becomes

$$
\mathscr{T}(\mathscr{U}) \leq \frac{1}{2 \eta} \operatorname{tr}(V) \operatorname{tr}(R)
$$

Thus, the performance bound (39) is independent of $\delta_{1}$. Furthermore, it is easy to check that $P_{\mathrm{T}}$ satisfies the equation

$$
0=A^{\mathrm{T}} P_{T}+P_{T} A+J^{\mathrm{T}} R J+R .
$$

We now present an alternative choice of $P_{0}(\Delta A)$.

Proposition 4.6. Let

$$
P=\left[\begin{array}{ll}
P_{11} & P_{12} \\
P_{12} & P_{22}
\end{array}\right], \quad R=\left[\begin{array}{ll}
R_{11} & R_{12} \\
R_{12} & R_{22}
\end{array}\right]>0
$$

satisfy (37) and let $P_{0}(\Delta A) \triangleq \mu I_{2}$, where

$$
\mu \triangleq \frac{\sqrt{\delta_{1}^{2}+\delta_{1}^{4}}}{2 \eta} \sqrt{\left(P_{22}-P_{11}\right)^{2}+\left(2 P_{12}\right)^{2}} .
$$

Then (9) and (10) are satisfied with $R_{0}=0$. Furthermore, the performance bound (15) is given by

$$
\mathscr{T}(\mathscr{U}) \leq \operatorname{tr}(P V)+\mu \operatorname{tr}(V) .
$$

Proof. Since $P \geq 0$ and $P_{0}(\Delta A) \geq 0, \Delta A \in \mathscr{Q}$, it follows that (10) is satisfied. Next, to show that (9) is true, recall that $\Omega_{0}(P)$ is given by equation (36). Therefore,

$$
\begin{aligned}
\Omega_{0} & (P)-\left[\left(A+\sigma_{1} J\right)^{\mathrm{T}} P_{0}+P_{0}\left(A+\sigma_{1} J\right)\right]-\sigma_{1}\left(J^{\mathrm{T}} P+P J\right) \\
& =\delta_{1}^{2}\left(-P+J^{\mathrm{T}} P J\right)-\mu\left(A^{\mathrm{T}}+A\right)-\sigma_{1}\left(J^{\mathrm{T}} P+P J\right) \\
& =2 \mu \eta I_{2}+\delta_{1}^{2}\left(-P+J^{\mathrm{T}} P J\right)-\sigma_{1}\left(J^{\mathrm{T}} P+P J\right) \\
& =2 \mu \eta I_{2}+S\left[\begin{array}{cc}
\lambda_{1} & 0 \\
0 & \lambda_{2}
\end{array}\right] S^{\mathrm{T}} \\
& =S\left[\begin{array}{cc}
2 \mu \eta+\lambda_{1} & 0 \\
0 & 2 \mu \eta+\lambda_{2}
\end{array}\right] S^{\mathrm{T}},
\end{aligned}
$$

where $\lambda_{1}=-\lambda_{2}=\sqrt{\sigma_{1}^{2}+\delta_{1}^{4}} \sqrt{\left(P_{22}-P_{11}\right)^{2}+\left(2 P_{12}\right)^{2}}$ are the eigenvalues of $\delta_{1}^{2}\left(-P+J^{\mathrm{T}} P J\right)-$ $\sigma_{1}\left(J^{\mathrm{T}} P+P J\right)$ and $S$ is a $2 \times 2$ orthogonal matrix. Choosing $\mu$ according to (42) implies that $2 \mu \eta+\lambda_{1} \geq 0$ and $2 \mu \eta+\lambda_{2} \geq 0$. Thus, (9) is satisfied. Finally, the performance bound (15) has the form

$$
\mathscr{T}(\mathscr{U}) \leq \operatorname{tr}\left[\left(P+P_{0}(\Delta A)\right) V\right]=\operatorname{tr}(P V)+\mu \operatorname{tr}(V) .
$$


Remark 4.7. As in $[3,4]$ the robust performance bounds (40) and (43) are only valid for constant uncertainty $\sigma_{1}$.

Before we present a numerical example, we shall illustrate some important aspects of $P$ given by equation (37). The analytical solution for (37) yields

$$
\begin{aligned}
& P_{11}+P_{22}=\frac{1}{2 \eta}\left(R_{11}+R_{22}\right), \quad P_{11}-P_{22}=\frac{1}{x}\left[\frac{\eta+\delta_{1}^{2}}{2}\left(R_{11}-R_{22}\right)-\omega R_{12}\right], \\
& 2 P_{12}=\frac{1}{\alpha}\left[\frac{\omega}{2}\left(R_{11}-R_{22}\right)+\left(\eta+\delta_{1}^{2}\right) R_{12}\right],
\end{aligned}
$$

where $\alpha \triangleq\left(\eta+\delta_{1}^{2}\right)^{2}+()^{2}$. For large $\delta_{1}$, it is easy to see that

$$
P_{11}-P_{22} \sim \frac{1}{2 \delta_{1}^{2}}\left(R_{11}-R_{22}\right), \quad 2 P_{12} \sim \frac{1}{\delta_{1}^{2}} R_{12}
$$

and

$$
\lim _{\delta_{1} \rightarrow \infty}\left[A_{1}^{\mathrm{T}}, P\right]=\lim _{\delta_{1} \rightarrow \infty}\left[\begin{array}{cc}
-2 P_{12} & P_{11}-P_{22} \\
P_{11}-P_{22} & 2 P_{12}
\end{array}\right]=0,
$$

which agrees with Lemma 3.9. Hence, $P_{11}-P_{22}$ and $P_{12}$ both approach zero as $\delta_{1} \rightarrow \infty$. These properties are the so-called equipartition (modal energy equilibration) and incoherence (modal decorrelation) phenomena $[17,20]$. Since

$$
\bar{\mu} \triangleq \lim _{\delta_{1} \rightarrow \infty} \mu=\frac{1}{2 \eta} \sqrt{\left(\frac{R_{11}-R_{22}}{2}\right)^{2}+R_{12}^{2}},
$$

the performance bound given by (43) approaches a ( finite) constant as $\delta_{1} \rightarrow \infty$. Furthermore, since $\lim _{\delta_{1} \rightarrow \infty} P_{11}=\lim _{\delta_{1} \rightarrow \infty} P_{22}=(1 / 4 \eta) \operatorname{tr}(R)$, it follows that

$$
\lim _{\delta_{1} \rightarrow \infty} \operatorname{tr}(P V)+\mu \operatorname{tr}(V)=\left(\frac{1}{4 \eta} \operatorname{tr}(R)+\bar{\mu}\right) \operatorname{tr}(V) .
$$

We now compare the performance bounds given by (39) and (43) for large values of $\delta_{1}$. Denoting $\mathscr{T}_{1}=\operatorname{tr}(V) \operatorname{tr}(P)$ and $\mathscr{T}_{2}=\operatorname{tr}(P V)+\mu \operatorname{tr}(V)$, it can be shown using $R_{12}^{2}<R_{11} R_{22}$ that

$$
\lim _{\delta_{1} \rightarrow \infty} \mathscr{T}_{1}-\mathscr{T}_{2}=\frac{\operatorname{tr}(V)}{2 \eta}\left[\frac{R_{11}+R_{22}}{2}-\sqrt{\left(\frac{R_{11}-R_{22}}{2}\right)^{2}+R_{12}^{2}}\right]=\frac{\operatorname{tr}(V)}{2 \eta} \lambda_{\min }(R)>0 .
$$

Finally, if $\operatorname{det} R=0$, then $\lim _{\delta_{1} \rightarrow \infty} \mathscr{T}_{1}=\lim _{\delta_{1} \rightarrow \infty} \mathscr{T}_{2}=(1 / 2 \eta) \operatorname{tr}(V) \operatorname{tr}(R)$.

\section{Numerical examples}

Example 5.1. Let us consider a lightly damped system with $\zeta=0.02, \omega_{n}=2, \eta=\zeta \omega_{n}, \omega=\sqrt{1-\zeta^{2}} \omega_{n}$,

$$
A=\left[\begin{array}{lr}
-\eta & \omega \\
-\omega & -\eta
\end{array}\right], \quad J=\left[\begin{array}{rr}
0 & 1 \\
-1 & 0
\end{array}\right],
$$

and let

$$
R=\left[\begin{array}{cc}
2 \beta & 0 \\
0 & 2
\end{array}\right]
$$


where $\beta>0$. For robust stability, we compare our result to the approach of [22]. For $R \neq 2 I_{2}$ we must use a congruence transformation in order to apply the theorem in [22]. Hence, we transform

$$
A^{\mathrm{T}} P+P A+R=0
$$

to obtain

$$
\hat{A}^{\mathrm{T}} \hat{P}+\hat{P} \hat{A}+2 I_{2}=0,
$$

where $\hat{A} \triangleq S^{-1} A S$, and $S$ is the congruence transformation matrix such that $S^{\mathrm{T}} R S=2 I_{2}$. As was mentioned in Remark 4.3, this system is robustly stable for all $\sigma_{1} \in \mathbb{R}$. This follows from [22] by taking $\beta=1$, that is, $R=2 I_{2}$, so that equation (45) has the solution $P=(1 / \eta) I_{2}$. Therefore, in the notation of [22], $P_{1} \triangleq \frac{1}{2}\left(J^{\mathrm{T}} P+P J\right)=0$, and thus the singular values of $P_{1}$ are all zero. As a result, the robust stability region is $\left|\sigma_{1}\right|<\infty$.

Now consider the case $\beta \gg 0$. Following the same procedure mentioned above, we have $\left|\sigma_{1}\right| \leq \delta_{1} \sim(2 / \omega \beta)\left(\eta^{2}+\omega^{2}\right)$ as $\beta \rightarrow \infty$. Thus, for large $\beta$ the approach of [22] becomes highly conservative. The reason for this conservatism is the similarity transformation of the skew-symmetric matrix $J$ which was effectively imposed by the choice $R \neq 2 I_{2}$. In the new basis, the matrix $J$ is transformed to $S^{-1} J S$, which is no longer skew-symmetric.

Example 5.2. Consider the same system in Example 5.1 except with

$$
R=\left[\begin{array}{ll}
2 & 1 \\
1 & 1
\end{array}\right]
$$

and for robust performance, let

$$
V=\left[\begin{array}{ll}
2 & 1 \\
1 & 1
\end{array}\right]
$$

First, the robust stability region found by using the same technique as in the previous example is $\left|\sigma_{1}\right|<1.37$, an extremely conservative result. As in the previous example, the reason for this conservatism is due to the similarity transformation of the skew-symmetric matrix $J$. In the new basis, the matrix $J$ is transformed to $S^{-1} J S$, which is no longer skew-symmetric.

Next, let us compare the robust performance bound given by equation (39) in Proposition 4.2 with the bound suggested by Bernstein and Haddad [3]. According to (39) the performance bound is $\mathscr{T}(\mathscr{U}) \leq(1 / 2 \eta) \operatorname{tr}(R)=98.50$, which is valid for all $\sigma_{1} \in \mathbb{R}$. In [3] the stability region and performance bound can be found by solving

$$
A^{\mathrm{T}} P_{A}+P_{A} A+A+R=0
$$

and by determining the values of $\sigma_{1}$ such that

$$
\sigma_{1}\left(A_{1}^{\mathrm{T}} P_{A}+P_{A} A_{1}\right) \leq \Lambda,
$$

where $A$ is a nonnegative-definite matrix. First, letting $A=k I_{2}$, where $k \geq 0$, it can be shown that the solution to equation (46) is $P_{A}=P+(k / 2 \eta) I_{2}$, where $P$ is the solution to (45) with

$$
R=\left[\begin{array}{ll}
2 & 1 \\
1 & 1
\end{array}\right]
$$

Therefore, we have the performance bound $\mathscr{T}(\mathscr{U}) \leq \operatorname{tr}(P V)+(k / 2 \eta) \operatorname{tr}(V)$ with robust stability region $\left|\sigma_{1}\right| \leq k / \lambda_{\max }\left(J^{\mathrm{T}} P+P J\right)$ (see Fig. 1). Alternatively, choosing $A=0.53 R$ yields the robust stability region $-2.57 \leq \sigma_{1} \leq 0.37$ which yields the symmetric stability region $\left|\sigma_{1}\right| \leq 0.37$. For this robust stability region the performance bound $\mathscr{T}(U) \leq 118.20$ (see Fig. 2). 


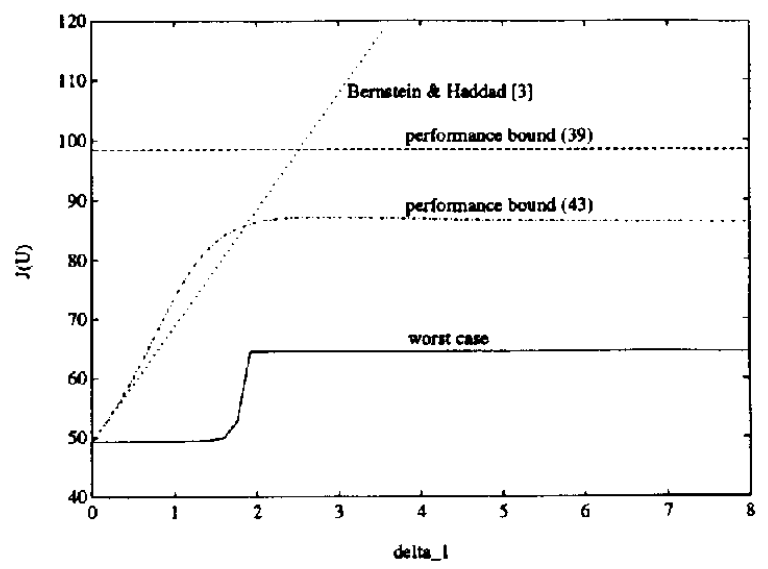

Fig. 1. Comparison of different robust performance bounds

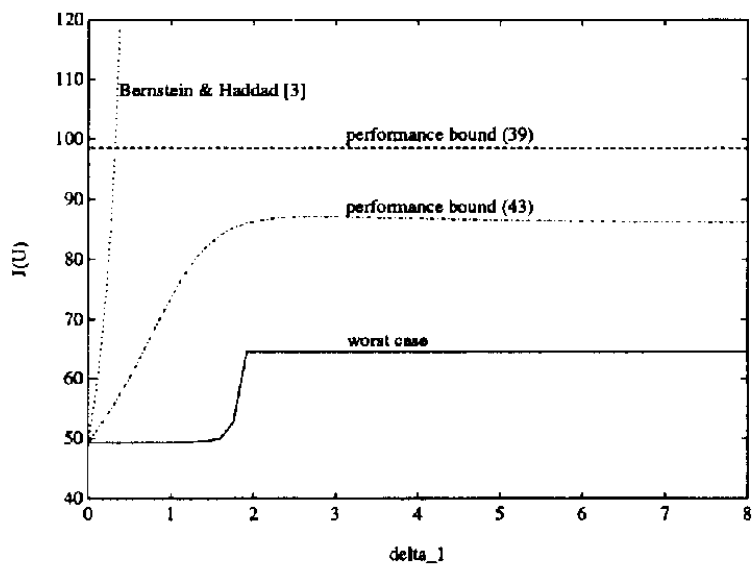

Fig. 2. Comparison of different robust performance bounds

\section{Discussion and conclusions}

As was shown in Propositions 4.2 and 4.6, the maximum-entropy-type Lyapunov functions correctly predict unconditional robust stability for arbitrary coordinates and thus, effectively, for an arbitrary state space basis. In addition, the performance bounds predicted by the maximum-entropy Lyapunov function are comparatively tight, even for large $\delta_{1}$, whereas the bound of [3] is extremely conservative and highly coordinate-dependent. The problem of choosing an appropriate basis may be relatively benign if robust stability analysis is performed independently of robust performance analysis. That is, for robust stability analysis one can arbitrarily choose the state space basis to produce the best estimate of the robust stability region without regard to robust performance. However, in the problem of robust controller synthesis the basis is not arbitrary but rather is dictated by the weighting matrices $V$ and $R$. Thus, the fact that the maximum-entropy-type Lyapunov functions provide robust stability and performance bounds that are only slightly affected by the choice of $V$ and $R$ appears to be a desirable feature for robust controller synthesis. This may explain the favorable results obtained in $[2,5,6,18,19]$. 


\section{Acknowledgment}

We wish to thank Jonathan How for noting Remark 4.5.

\section{References}

[1] D.S. Bernstein, Robust static and dynamic output-feedback stabilization: deterministic and stochastic perspectives, IEEE Trans. Automat. Control 32 (1987) 1076-1084.

[2] D.S. Bernstein and S.W. Greeley, Robust controller synthesis using the maximum entropy design equations, IEEE Trans. Automat. Control 13 (1986) 362-364.

[3] D.S. Bernstein and W.M. Haddad, Robust stability and performance analysis for linear dynamic systems, IEEE Trans. Automat. Control 34 (1989) 751-758.

[4] D.S. Bernstein and W.M. Haddad, Robust stability and performance analysis for state space system via Quadratic Lyapunov bounds, SIAM J. Matrix Anal. Appl. 11 (1990) 239-271.

[5] D.S. Bernstein and D.C. Hyland, The optimal projection/maximum entropy approach to designing low-order, robust controllers for flexible structures, in: Proc. IEEE Conf. Dec. Contr., Fort Lauderdale, FL (1985) 745-752.

[6] D.S. Bernstein and D.C. Hyland, The optimal projection approach to robust, fixed-structure control design, in: J.L. Junkins, ed., Mechanics and Control of Space Structures (AIAA, New York, 1990) 287-293.

[7] J.W. Brewer, Kronecker products and matrix calculus in system, IEEE Trans. Circuits and Systems 25 (1978) $772-781$.

[8] S.L. Campbell and C.D. Meyer Jr., Generalized Inverse of Linear Transformation (Pitman, New York, 1979).

[9] M. Cheung and S. Yurkovich, On the robustness of MEOP design versus asymptotic LQG synthesis, IEEE Trans. Automat. Control 33 (1988) 1061-1065.

[10] E.G. Collins Jr., J.A. King and D.S. Bernstein, Robust control design for the benchmark problem using the maximum entropy approach, in: Proc. Amer. Contr. Conf., Boston, MA (1991) 1935-1936.

[11] E.G. Collins Jr., et al., High performance accelerometer-based control of the mini-MAST structure at Langley Research Center, NASA Contractor Report 4377, 1991.

[12] A. Gruzen, Robust reduced order control of flexible structures, C.S. Draper Laboratory Report CSDL-T-900, 1986.

[13] A. Gruzen and W.E. van der Velde, Robust reduced order control of flexible structures using the optimal projection/maximum entropy design methodology, in: AIAA Guidance, Natigation, and Control Conf., Williamsburg, VA (1988).

[14] W.M. Haddad and D.S. Bernstein, Robust stabilization with positive real uncertainty: beyond the small gain theorem, Systems Control Lett. 17 (1991) 191-208.

[15] W.M. Haddad and D.S. Bernstein, Parameter-dependent Lyapunov functions, constant real parameter uncertainty, and the Popov criterion in robust analysis and synthesis, in: Proc. IEEE Conf. Dec. Contr., Brighton (1991) 2274-2279 (Part I), 2632-2633 (Part II).

[16] N. W. Hagood IV and E.F. Crawley, Cost averaging techniques for robust control of parametrically uncertain system, MIT SERC Report \#9-91, 1991.

[17] S.R. Hall, D.G. MacMartin and D.S. Bernstein, Covariance averaging in the analysis of uncertain systems, IEEE Trans. Automat. Control, to appear.

[18] D.C. Hyland, Maximum entropy stochastic approach to controller design for uncertain structural systems, in Proc. American Control Conf., Arlington, VA (1982) 680-688.

[19] D.C. Hyland and A.N. Madiwale, A stochastic design approach for full-order compensation of structural systems with uncertain parameters, in: Proc. AIAA Guidance and Control Conf.. Albuquerque, NM (1981) 324-332.

[20] R.H. Lyon, Statistical Energy Analysis of Dynamical Systems: Theory and Applications (MIT Press, Cambridge, MA, 1975).

[21] W.M. Wonham, Linear Multivariable Control: A Geometric Approach (Springer, New York, 1974).

[22] K. Zhou and P.P. Khargonekar, Stability robustness bounds for linear state-space models with structured uncertainty, IEEE Trans. Automat. Control 32 (1987) 621-623. 